\title{
Dialysis and the Elderly: An Underestimated Problem
}

\author{
Michele Buemi Antonio Lacquaniti Davide Bolignano Valentina Donato \\ Maria Rosaria Fazio Susanna Campo Giuseppe Coppolino Alessio Sturiale \\ Chair of Nephrology, Department of Internal Medicine, University of Messina, Messina, Italy
}

\section{Key Words}

Uremia $\cdot$ Dialysis $\cdot$ Elderly $\cdot$ Vascular access $\cdot$ Quality of life

\begin{abstract}
In developed countries, the incidence of end-stage renal failure is constantly increasing, and uremia will soon be a disease typically found in mature and elderly adults. Almost invariably, the physical condition of the elderly patient with terminal uremia is extremely poor, and therapeutic approach complex. Frequent co-morbidity, treatment with many different drugs, the high risk of iatrogenic damage, advanced age and socio-environmental conditions further complicate the management of these patients. While replacement therapy may become necessary, peritoneal dialysis may have advantages over hemodialysis. Peritoneal dialysis causes less hemodynamic stress, does not necessitate vascular access and allows mobility, although it incurs a high incidence of peritonitis and vascular disease. Where hemodialysis is the only feasible treatment, procedures used for vascular access are frequently followed by several complications, representing an important cause of morbidity and hospitalization. In addition, even if it may improve the patient's quality of life, vascular condition, intradialytic hypotension, heart disease, intestinal bleeding and amyloidotic arthropathy are critical aspects of dialysis in the elderly patient. Therefore, particular attention from clinicians and administrators is required and the best possible strategies must be identified in order to provide effective and appropriate services to address these special patients' needs.

Copyright $\odot 2008$ S. Karger AG, Basel
\end{abstract}

\section{Introduction}

In developed countries, the incidence of end-stage renal failure is constantly increasing, and uremia will soon be a disease typically found in mature and elderly adults. In recent years, this trend has come to involve both elderly and old-old ( $>75$ years) patients, a particularly relevant increase being found in the latter category. In the United States, the incidence of renal failure in patients over 75 years of age has markedly increased in recent years [1].

It is therefore possible that the requests for replacement therapy in uremic patients, particularly the elderly undergoing hemodialysis, will grow by $4.5-6 \%$ until the year 2010, and will not plateau for at least another 25 years [2]. In Italy, the National Dialysis Registry reports even higher incidence and prevalence ratios (24 and 17\%, respectively) in subjects aged over 75 years. The overall clinical status of elderly uremic patients on chronic dialysis therapy is frequently very poor, and the duration of replacement therapy, usually lasting several years, may further compromise their condition, making geriatric care mandatory. Chronic heart failure, acute coronary syndromes, myocardial infarction, and its immediate and late complications, play a role in the clinical picture of the uremic dialytic patient, and may also play a part in accelerating atherosclerotic processes which, in turn, are linked to the underlying chronic renal failure [3].

Patients with uremia also tend to have acute proteic and energetic malnutrition, notwithstanding the con-

\section{KARGER}

Fax +4161306 1234

E-Mail karger@karger.ch

www.karger.com

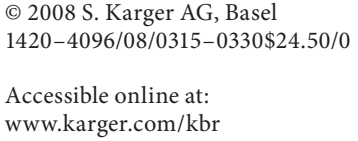

Prof. Michele Buemi

Via Salita Villa Contino, 30

IT-98100 Messina (Italy)

Tel. +39090 221 2265, Fax +390902935162

E-Mail buemim@unime.it 
stant dietary advice usually given in such cases. Among the many clinical consequences (i.e. immune system deficiencies, infectious complications, drug transport and metabolic impairment, reduced anti-oxidative potential), a reduced muscular-skeletal mass is usually markedly present [4].

The latter, which is the main factor in the so-called wasting syndrome, is easily distinguished from age-related sarcopenia, due to its rapid onset and etiology.

Combined, the two catabolic processes severely compromise the patients' mobility, thus further exacerbating their condition.

Loss of autonomy, the immediate consequence of hypomotility, may also contribute to a clinical deterioration, thus worsening the elderly patient's prognosis. Severe loss of autonomy can call for an interruption in treatment, which is usually scheduled on a 3-weekly basis. Uremia decreases glucose tolerance which, in turn, can cause diabetes mellitus, known to lead to diabetic nephropathy in a large percentage of patients; the latter condition, which is complex and multiform, leads to advanced renal failure, dialysis becoming necessary.

Geriatric and uremic patients may therefore have several features in common, including malnutrition, comorbidities, hypomotility, and cardiovascular and infectious diseases [5].

Patients in both categories may also have depression, cognitive impairment, ranging from mild alterations to severe dementia, and a poor economic status and physical dependency and, as their condition is longstanding and incurable, they are often 'institutionalized'. In two studies conducted by British and Canadian General Practitioners (GPs), it has been found that the older and the more severely compromised the patients, the less often are they referred to a nephrologist. In recent years, it was found among clinicians interviewed that $19 \%$ of GPs and $44 \%$ of North American internists decided not to consult a nephrologist when dealing with terminal chronic renal failure patients [6]. Joly et al. [7, 8] demonstrated that $34 \%$ of elderly patients are referred to nephrologists four months before beginning dialysis.

\section{Dialysis or Conservative Therapy?}

It is important to consider whether or not age alone can be considered an ethically acceptable criterion for access to dialysis. Data reported in the literature suggest that the factors most often leading to the renouncement of dialysis are dementia, acute malnutrition, cancer and/ or loss of autonomy. In several studies it has, in fact, been demonstrated that the start of dialysis is frequently considered questionable in patients with cognitive disorders and, less frequently, it may be denied to patients with reduced physical autonomy, weight loss and/or co-morbidity $[9,10]$.

Furthermore, in a retrospective study conducted in Japan on a cohort of 152 elderly patients with chronic renal failure, it was found that advanced age $(>80)$ and cognitive disorders were the two most important factors in preferring conservative therapy to dialysis, whereas social marginalization, malnutrition and physical dependency played a minor role [11].

The use of a conservative approach may occur secondary to a medical decision or to the patient's wish to avoid dialysis. Conservative therapy calls for a close study of the patient's symptoms together with the careful management of anemia, water and salt overload, hydroelectric imbalance, pain and/or malnutrition. Psychosocial problems should be dealt with by psychologists and social assistants. In a cohort of 43 uremic patients aged 80 years, Joly et al. [12] reported a survival of about 9 months following conservative treatment. Fliss et al. [13] underline the relevance of co-morbidites in choosing between conservative therapy and dialysis. However, the percentage of early deaths due to arrhythmia (34\%) or pulmonary edema $(24 \%)$ in patients on conservative therapy indicate that dialytic treatment can lead to better survival outcome.

\section{Dialytic Treatment: Which Is the Best Choice?}

Hemodialysis requires the elderly patient to be admitted to a day-care service (hospital or clinic), whereas peritoneal dialysis (PD) can be provided in the patient's home. The choice between the two techniques is often related to the availability or unavailability of either procedure, local competency and the patient's wishes. However, if an elderly patient is referred to the nephrologist with latestage disease, hemodialysis is usually the only therapeutic approach possible.

Several age-related physiological changes may influence the decision on therapy: for example, a silent or symptomatic reduction in cardiovascular reserve secondary to atherosclerosis or baroreceptor dysfunction, loss of pulmonary function, immune system imbalance, infectious diatheses, metabolic disturbances, osteoporosis, altered protein metabolism, drugs, severe malnutrition and decreased glucose tolerance. 
However, the possible advantages of hemodialysis with respect to PD are still widely debated. In a recent study from the Netherlands, nephrologists were asked to indicate the most important reason for dialysis modality selection. $83 \%$ of medical and $97 \%$ of social contraindications were for $\mathrm{PD}$, and the most frequently mentioned contraindication was the expected inability of patients to perform exchanges themselves [14].

In elderly patients, $\mathrm{PD}$ causes less hemodynamic stress, does not necessitate vascular access, allows patients mobility and provides them with the sense that their disease is under control; equally important, it maintains residual diuresis. Furthermore, hemodialysis is associated with relevant fluctuations in cerebral performance during the course of, and during, the dialysis session, while cerebral performance is stable in patients on PD [15]. Yet, there are contraindications to PD in both elderly and younger patients: inadequate functioning of the peritoneal membrane, inoperable hernia, chronic peritoneal access making this approach unfeasible. Relative contraindications are: recurrent pancreatitis, chronic back pain, recent insertion of aortic prosthesis, severe peripheral vascular disease and chronic diverticulitis. PD in the elderly patient has potential disadvantages in the presence of depression and physical or mental debility if there is no guarantee that the procedure can be performed in the context of adequate associated patient care. Psychosocial problems (cognitive problems, depression, non compliance, social isolation and dependency on help) are barriers that can be overcome provided that proper support and assistance are offered to the PD patient at home [16]. Moreover, pain, malnutrition and frequent peritonitis linked hospitalization may be tolerated less by elderly than by younger patients. There is, however, growing evidence that the use of community nurses to assist with PD and the introduction of a program for assisted peritoneal dialysis (aPD) targeting frail, elderly patients may enable more elderly patients to have their PD treatment at home [17]. Verger et al. [18] have recently shown that aPD patients assisted by a non-trained private nurse have a significantly higher risk of peritonitis compared with family-assisted patients. This suggests that all PD training and retraining of PD patients, relatives, or assistants should be provided by dedicated expert PD nurses from the PD unit [19]. The ever-increasing number of older and sicker patients with end-stage kidney disease who are initiating chronic dialysis treatment is a major challenge facing every dialysis unit and every dialysis modality presently. Increasing the use of aPD will generally improve the lifestyle of the fit elderly and will allow more patients to remain on PD in their own environment for a longer period of time. We believe that, in the future, aPD will prove to be a safe and feasible complementary alternative to in-center hemodialysis for the growing group of frail, elderly patients with end-stage kidney disease. Given the many difficulties and challenges of running an aPD program, as discussed previously, it is evident that the success of such a program is critically dependent on a well-organized multidisciplinary team of dedicated expert renal nurses, assistants, nephrologists, dieticians, social workers, and others. In addition, any malnutrition may be exacerbated by dialysis-related anorexia, nausea and protein-wasting. Elderly patients tend to eat less than their younger counterparts, and economic problems (inertia and apathy, depression, nausea, reduced mobility and drug therapy) may all contribute to exacerbating their condition. In order to ensure the adequate intake of proteins, calories, vitamins and minerals, it is advisable to plan tailored dietary schedules for patients on PD. Although the incidence of complications from PD in the elderly is similar to that in younger patients, in the former category hospitalization is required more frequently, and for longer periods of time than in the latter because the incidence of peritonitis and vascular disease is higher. With respect to younger adults, elderly patients, mainly due to their reduced food intake, tend to have smaller inter-dialytic weight gain, lower predialytic potassium levels and a better compliance both to therapy and to diet prescriptions. If patients with degenerative or atherosclerotic processes are excluded, they also tend to be more emotionally stable than their younger counterparts. Dialytic sessions can also be considered an occasion for socialization, an aspect that is particularly important in the case of the elderly, many of whom suffer from loneliness [20]. Older people often do not want to be involved in clinical decisions about their treatment. Ahmed et al. [21] have analyzed the opinions of elderly people on the acceptance and toleration of renal replacement therapy using a questionnaire based on a clinical vignette. From this study, it emerged that elderly people want dialysis therapy. Also, Mead et al. [22] demonstrated that elderly patients are in favor of high-technology medical interventions and are willing to put up with considerable interference with their life-style, providing there is reasonable symptom relief. Steuer [23] found that about $80 \%$ of dialysis patients over 80 years would recommend dialytic treatment to patients of the same age, while only $12.5 \%$ would suggest to refuse this kind of therapy. 
Fig. 1. Treatment of malnutrition in uremic elderly: a brief flow-chart.

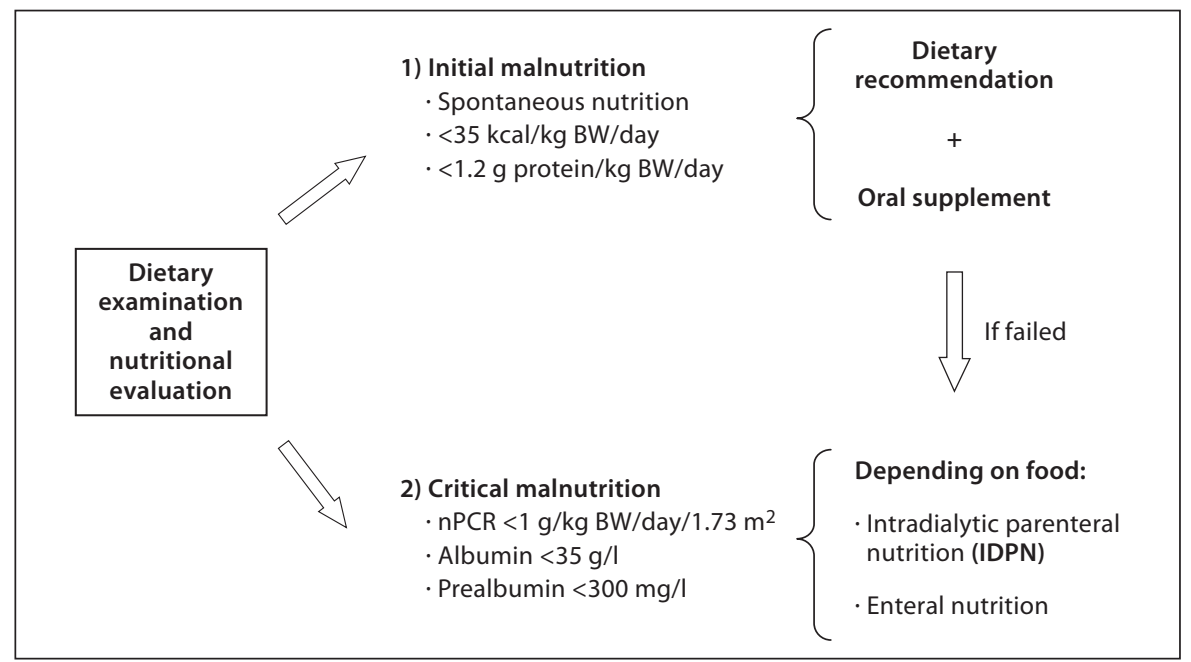

\section{Nutritional Approach in Uremic Elderly}

Renal failure can worsen the condition of malnutrition often observed in elderly patients because of the onset of symptoms like nausea and anorexia and the protein loss in dialysate. The food intake in elderly people is generally reduced with respect to young patients because of their economic and psychological conditions, movement impairment and drug consumption.

However, the protein intake in elderly patients with CKD in conservative therapy should not be different from that of a normal adult (0.7-0.8 g/kg BW/day) [24].

In fact, both Italian and English nephrologists agree in recommending a protein intake of $0.8 \mathrm{~g} / \mathrm{kg}$ BW/day and a caloric intake of $35 \mathrm{kcal} / \mathrm{kg}$ BW/day as the upper limit [25].

The Kidney Dialysis Outcome Quality Initiative (KDOQI) recommends a 3-monthly check of the nutritional status using as markers body weight, albuminemia, protein consumption. A caloric intake of $30-35 \mathrm{kcal} / \mathrm{kg} /$ day is recommended for patients over 60 years who do not practice intense physical activity.

More active subjects should introduce the same caloric amount as younger patients ( $>35 \mathrm{kcal} / \mathrm{kg} /$ day). Malnutrition, in fact, represents a negative prognostic index for the evolution of renal disease and the onset of clinical signs is an indication for dialysis start.

Brunori and coworkers $[26,27]$ have studied patients older than 70 years with a GFR of $5-7 \mathrm{ml} / \mathrm{min} / 1.73 \mathrm{~m}^{2}$ and cardiac ejection fraction greater than $30 \%$. These patients were separated into two groups: the first treated with a SVLPD (diet group: a very-low-protein diet supplemented with essential amino acids and keto analogues), and the second receiving maintenance dialysis (dialysis group: started dialysis therapy in the case of malnutrition, intractable fluid overload, hyperkalemia, or appearance of uremic symptoms). They have demonstrated that maintenance dialysis therapy should be initiated when renal function decreases below the level at which it is recommended. sVLPD is safe when postponing dialysis treatment by a median of 10.7 months (range 1-58 months).

In patients undergoing dialysis, especially in the elderly, an insufficient protein and calories intake is associated with an increased mortality and morbidity risk. Death due to malnutrition is not rare among patients over 70 years: this phenomenon has been explained by the reduced biocompatibility of dialysis equipment and the production of stress hormones during the session [28]. Another important question concerning elderly patients which needs to be considered is the irregular food intake depending on dialytic rhythm. A study by Bellizzi et al. [29] demonstrated an increase of about $10 \%$ in food intake during the days in which the patients do not undergo hemodialysis, while in some patients the food intake is reduced by about $40 \%$ at the 7 th day (the last day of the longest interdialytic period). In conclusion, the nutritional status of elderly CKD patients is still a problematic issue and the therapeutic approach has not been substantially improved in the last 20 years. Recently, new treatment options have been proposed and they are being tested in large clinical trials in Europe [30]. A systematic surveillance of the nutritional status of these patients and an early identification of signs of malnutrition allows quick intervention in order to stop the vicious circle which leads to denutrition (fig. 1). 


\section{Vascular Access in the Elderly Patient on Dialysis}

Vascular access procedures and related complications in patients on chronic dialysis are an important cause of morbidity and hospitalization. Patients aged over 65 years have a particularly high incidence of comorbidity factors (diabetes mellitus, atherosclerosis, heart failure); the appropriate decision regarding time and type of permanent access therefore is of crucial importance in this patient category. Latos et al. [31] found that only $14 \%$ of 173 patients aged over 65 years on dialysis had an arterovenous fistula (AVF), while $52 \%$ had a prosthesis and $34 \%$ a central catheter. When compared with younger populations, elderly patients are found to have fewer fistulas and more catheters. In a series of 81 dialyzed patients aged over 60 years, direct AVF was achieved in $61.6 \%$ of cases $(85 \%$ of which were radial) and $38.4 \%$ had autologous interpositioning of the saphenous vein. The duration of AVF utilization was 25 months, less than in the group of younger patients (35 months). The transplanted vessels had a rate of utilization at 3 years of 57\% (autologous) and 12\% (homotransplanted). The advantages of prostheses are widely recognized in the elderly: they can be used more speedily, are easier to pierce and technically less tricky to achieve, and are used, in particular, in subjects with small lumen vessels or vascular disease. In the US, a significantly greater number of prostheses than AVF are used. Chertow [32] maintains that this increase in the use of these surgical techniques should be inverted in favor of the creation of fistulae, in order to limit morbidity directly related to vascular access. Elcheroth et al. [33] observed that levels of functionality at 4 years are more than $50 \%$ for fistulae to the arm, and prefer this approach to prosthetic forearm bypass. An adequate fistula is equally important for obtaining correct clearance of the dialyzer $(\mathrm{Kt} / \mathrm{V})$. According to experience gained by Mandolfo et al. [34], who used a radiocephalic fistula in elderly patients, the access flow obtained was lower in the elderly than in younger patients (although the difference was not significant, at $738 \pm 350 \mathrm{vs.} 892 \pm 491 \mathrm{ml} / \mathrm{min})$. The authors underlined the need, particularly in malnourished patients, to prescribe an adequate dialytic dose $(\mathrm{Kt} / \mathrm{V}$ $>1.3$ ) identical to that in younger patients. In functional terms, the duration of the fistulae can be significantly prolonged by performing percutaneous angioplasty: Burger et al. [35] reported an improvement in the levels of permeability of distal fistulae of $65-80 \%$ at 2 years with the use of endoluminal percutaneous angioplasty.

Currently, interventional radiology can be utilized as a first intent to treat stenosis and thrombosis, surgery be- ing reserved for cases in which this technique fails, such as aneurysm, sepsis and hyper afflux. These conditions, however, call for the planning of a good quality vascular access. In elderly uremic subjects, the arteries are profoundly altered by arteriosclerosis and atherosclerosis and the accelerated process of aging typical of renal failure. Veins are often thin, with lumens that are altered due to previous blood sampling. Although, in expert hands, the success rate can be high, classic AVF of the wrist, which is often difficult to achieve, can be replaced by proximal AVF. Berardinelli et al. [36] have demonstrated that the initial failure occurs in $20 \%$ of AVF cases but only $1.8 \%$ of omeral cases. Satisfactory functioning at 1 year is 60 and $92 \%$, respectively, and 57 and $78 \%$ at 3 years. The incidence of thrombosis is very low. The above accesses, however, incur a risk of the so-called acute or chronic stealing syndrome, with a poor downstream vascular circulation, occurring above all in diabetics. The possibility of heart failure should also be considered. Vascular prostheses involve exposure to the same complications as those occurring in the adult uremic subjects, but infection is more frequent. Among the factors that can compromise the functioning of the vascular access, cardiovascular tolerance, greatly compromised in the elderly subject on dialysis, plays an important role. Finally, therapy for anemia using erythropoietin can cause an increase in blood viscosity that, according to some authors, is associated with a greater risk of thrombosis in elderly patients with AVF, as well as with protheses [37]. These issues can thus lead to a reduced perception of the need to create early vascular access in the elderly patient. This also explains why the probability of utilizing an AVF as the first type of vascular access diminishes with age [38]. Central venous catheters offer an effective, rapid and simple solution to the problem of transitory or permanent vascular access. Age does not modify their duration, which is $50 \%$ at 3 years in specialized centers. The most frequent complication, however, is infection, present in $10 \%$ of the cases, followed by thrombosis or poor functioning [39].

\section{Prognosis and Quality of Life}

According to recommendations made jointly by the American Society of Nephrology (ASN), elderly patients in the preterminal stages and/or their families should receive detailed information regarding life expectancy before dialysis is considered; the decision should be taken together with the physician [40]. The nutritional param- 
eters, including albumin, prealbumin and body mass index (BMI), are of great importance in establishing the elderly patient's prognosis: the course of dialysis is greatly influenced by the number of co-morbidities present at its initiation, and their severity. The age factor is of evident prognostic value, but when the effects of co-morbid factors are taken into consideration, age loses its impact. Therefore, the clinical and multidisciplinary evaluation of each single case is of fundamental importance, while taking into account the quality of life to be expected while the patient is on dialysis.

Numerous retrospective studies have evaluated the quality of life by utilizing indirect markers, such as the duration of hospitalization and Karnosky's functional score: the results are not as satisfactory as those observed in the general population. If, on the other hand, one utilizes the indices of patient satisfaction regarding his/her quality of life and the perception of dialysis by the elderly patient, the data obtained are promising. Lamping et al. [41], in a large cohort of elderly patients on dialysis, revealed that their perception of the quality of 'physical' life is inferior to that of the general population of the same age, whereas the score for quality of 'mental' life was the same as that in control subjects. The subjective nature of the methods utilized to evaluate quality of life compromised the uniformity of results; however, the elderly population on dialysis was more satisfied with its health status than the younger population, in spite of their greater morbidity with respect to the younger population. While the quality of life is sometimes unexpectedly good, the vascular condition, intra-dialytic hypotension, heart disease, intestinal bleeding and amyloid artropathy are critical aspects of dialysis in the elderly patient.

\section{Conclusions}

When a patient discovers that he has chronic renal failure calling for dialysis, he suffers a tremendous shock. As stated by an American psychiatrist [42], the patient on replacement therapy experiences three different phases: (1) so-called 'falling in love with dialysis', correlated with the fact that the patient associates dialysis with an improvement in quality of life; (2) a progressive disappointment, this phase representing a period of increased risk, and (3) a slow adaptation to dialysis - with, in the elderly patient, an inexorable decline toward depression.

The geriatric uremic patient is usually over 80 years old, has more than one chronic, progressive disease, which is frequently incurable, and often has problems concerning his mental health and functional autonomy, frequently living in problematic socioenvironmental conditions. This type of patient is at a high risk of invalidity and disability.

The elderly patient with terminal uremia who undergoes dialysis exemplifies the complex medical care often required by members of the aging population: he is almost always a complex and fragile patient. Frequent comorbidity, treatment with many different drugs, the high risk of iatrogenic damage, the state of advanced age, and the socio-environmental conditions, often critical factors, make the physical condition of elderly dialysis patients particularly precarious. Therefore, the approach used for them cannot hinge entirely upon traditional therapy, a holistic approach based upon a multidimensional evaluation being required. This calls for particular attention from clinicians, administrators and politicians: the best possible strategies must be identified so as to provide effective and appropriate services to these special patients' needs.

Considering the increasing incidence of end-stage renal disease in elderly patients, the economic impact of dialytic treatment in these subjects is becoming a relevant social problem. Nevertheless, the effects of chronic substitutive renal therapy are encouraging in terms of survival and life quality, so it should be avoided only in case of specific contraindications such as severe dementia and cancer. Nevertheless, many old patients do not undergo hemodialysis because of their social condition or because of the late referral to a nephrologist. In fact, an early nephrological evaluation allows to improve conservative therapy and dialytic survival of these patients.

Nephro-geriatric medicine has opened new important fields of clinical research, such as life quality evaluation, control of cognitive alterations, palliative therapies and ethical problems that need a multidisciplinary intervention.

The elderly are deserving of respect for what they have been, for the contributions they have made, and for the example they can give, thanks to their wisdom and dignity. Is it not, therefore, our duty to make every possible effort to give them a decent quality of life? 


\section{References}

$>1$ USRDS: Part II. Incidence and prevalence of ESRD. Am J Kidney Dis 1999;34:S40-S50.

$\checkmark 2$ Wolfe RA, Held PJ, Hulbert-Shearon TE, et al: A critical examination of trends in outcomes over the last decade. Am J Kidney Dis 1998;32:S9-S15.

3 Parry RG, Crowe A, Stevens JM, et al: Referral of elderly patients with severe renal failure: questionnaire survey of physicians. BMJ 1996;313:466

4 Aparicio M, Cano N, Chauveau P, et al: Nutritional status of haemodialysis patients: a French national cooperative study. French Study Group for Nutrition in Dialysis. Nephrol Dial Transplant 1999;14:16791686.

5 McDonald S: Dialysis outcome among the elderly: is it worthwhile? J Am Soc Nephrol 2004, p 23 (abstract SA-PO389).

6 Sekkarie M, Cosma M, Mendelssohn D: Non-referral and non-acceptance to dialysis by primary care physicians and nephrologists in Canada and the United States. Am J Kidney Dis 2001;38:36-41.

$>7$ Joly D, Anglicheau D, Alberti C, et al: Octogenarians reaching end-stage renal disease: cohort study of decision-making and clinical outcomes. J Am Soc Nephrol 2003;14:10121021.

8 Jungers P, Joly D, Nguyen-Khoa T, et al: Continued late referral of patients with chronic kidney disease. Causes, consequences, and approaches to improvement. Presse Méd 2006;35:17-22.

7 Mignon F, Michel C, Viron B, et al: Endstage renal diseases in patients 75 and over: a new medical, socio- economical and ethical challenge. Eur J Med 1992;1:302-307.

$\checkmark 10$ Holley JL, Foulks CJ, MOSS AH: Nephrologists' reported attitudes about factors influencing recommendations to initiate or withdraw dialysis. J Am Soc Nephrol 1991;1: 1284-1288.

-11 Fujimaki H, Kasuya Y, Kagami S, et al: Acceptance of dialysis therapy in elderly patients with chronic renal failure. Nippon Ronen Igakkai Zasshi 2003;40:41-46.

$>12$ Joly D: Dialysis therapy for end stage renal disease in octogenarians. Rev Prat 2005;55: 2255-2262.

13 Murtagh FE, Marsh JE, Donohoe P, et al: Dialysis or not? A comparative survival study of patients over 75 years with chronic kidney disease stage 5 . Nephrol Dial Transplant 2007;22:1955-1962.

14 Jager KJ, Korevaar JC, Dekker FW, et al: The effect of contraindications and patient preference on dialysis modality selection in ESRD patients in The Netherlands. Am J Kidney Dis 2004;43:891-896.
15 Williams MA, Sklar AH, Burright RG, et al: Temporal effects of dialysis on cognitive functioning in patients with ESRD. Am J Kidney Dis 2004;43:705-711.

16 Quinn RR, Oliver MJ: Is assisted peritoneal dialysis an alternative to in-center hemodialysis. Perit Dial Int 2006;26:650-653.

17 Povlsen JV, Ivarsen P: Assisted automated peritoneal dialysis (AAPD) for the functionally dependent and elderly patient. Perit Dial Int 2005;25(suppl 3):S60-S63.

18 Verger C, Duman M, Durand PY, et al: Influence of autonomy and type of home assistance on the prevention of peritonitis in assisted automated peritoneal dialysis patients: an analysis of data from the French Language Peritoneal Dialysis Registry. Nephrol Dial Transplant 2007;22:1218-1223.

$\checkmark 19$ Bernardini J, Price V, Figueiredo A: ISPD guidelines/recommendations 2006: peritoneal dialysis patient training. Perit Dial Int 2006;26:625-632.

20 Alloatti S, Manes M, Gaiter AM, et al: La dialisi nel paziente anziano G Ital Nefrol 2002; 19:622-629.

21 Ahmed S, Addicott C, Qureshi M, Pendleton N, Clague JE, Horan MA: Opinions of elderly people on treatment for end-stage renal disease. Gerontology 1999;45:156-159.

22 Mead GE, Pendleton N, Pendleton DE, Bent N, Rabbit P, Horan MA: High technology medical interventions: what do the elderly want? J Am Geriatr Soc 1997;45:1409-1411.

23 Steuer G: Der Dialysepatient im IX. Dezennium; Inauguraldiss, Freie Universität Berlin, 1997.

24 Rand WM, Pellet PL, Uoung VR: Meta-analysis of nitrogen balance studies for estimating protein requirements in healthy adults. Am J Clin Nutr 2003;77:109-127.

25 Locatelli F, Valderràbano F, et al: The management of chronic renal insufficiency in the conservative phase. Nephrol Dial Transplant 2000;15:1529-1534.

26 Brunori G, Viola BF, Parrinello G, et al: Efficacy and safety of a very-low-protein diet when postponing dialysis in the elderly: a prospective randomized multicenter controlled study. Am J Kid Dis 2007;49:569580.

27 Maiorca R, Brunori G, Gregorini G, et al: Very low protein diet in elderly ESRD patients prolongs residual renal function, improves survival and dialysis can be started late. J Am Soc Nephrol 1998;9:154A.

28 Bohe J, Rennie M: Muscle protein metabolism during haemodialysis. J Renal Nutr 2006;16:3-16.
29 Bellizzi V, Di Iorio BR, Terracciano V: Daily nutrient intake represents a modifiable determinant of nutritional status in chronic haemodialysis patients. Nephrol Dial Transplant 2003; 18:1874-1881.

30 Locatelli F, Fouque D, Heimburger O, Drüeke TB, Cannata-Andía JB, Hörl WH, Ritz E: Nutritional status in dialysis patients: a European consensus Nephrol Dial Transplant 2002; 17:563-572.

31 Latos DL: Chronic dialysis in patients over 65. J Am Soc Nephrol 1996;7:637-646.

32 Chertow G: Grafts vs. fistulas for hemodialysis patients, equal access for all? J Am Med Assoc 1996;276:1343-1345.

33 Elcheroth J, de Pauw L, Kinnaert P: Elbow arteriovenous fistulas for chronic haemodialysis. Br J Surg 1994;81:982-984.

34 Mandolfo S, Bucci R, Ravani P, et al: Dialysis prescription in elderly hemodialysis patients Renal Unit, Ospedale Maggiore, Lodi - Italy. J Vasc Access 2000;1:139-143.

35 Burger H, Kluchert BA, Kootstra G, et al; Survival of arteriovenous fistulas and shunts for haemodialysis. Eur J Surg 1995;161:327334.

36 Berardinelli L, Vegeto A: lessons from 494 permanent accesses in 348 haemodialysis patients older than 65 years of age: 29 years of experience. Nephrol Dial Transplant 1998; 13(suppl 7):S73-S77.

$>37$ Bonforte G, Zerbi S, Pasi A, et al: Distal arteriovenous fistulas in elderly hemodialysis patients Nephrology and Dialysis Unit, Desio Hospital, Desio, Milan - Italy. J Vasc Access 2000;1:144-147.

38 Limido A, Cantù P: Vascular access for chronic haemodialysis in elderly patients: the Lombardy experience. Nephrology and Dialysis Unit, S. Antonio Abate Hospital Gallarate, Varese - Italy. J Vasc Access 2000; 1:129-133.

39 Brunori G, Verzelletti F, Zubani R, et al: Which vascular access for chronic hemodialysis in uremic elderly patients? Institute and Division of Nephrology, Spedali Civili, University of Brescia, Brescia - Italy. J Vasc Access 2000;1:134-138.

40 Nephrology RPA/ASN: Shared decision making in the appropriate initiation of and withdrawal from dialysis. Clinical Practice Guideline Number 2. Rockville, 2000.

41 Lamping DL, Constantinovici N, Roderick P, et al: Clinical outcomes, quality of life, and costs in the North Thames dialysis study of elderly people on dialysis: a prospective cohort study. Lancet 2000;356:1543-1550.

42 Levy NB: Psychiatric considerations in the primary medical care of the patient with renal failure. Adv Ren Replace Ther 2000;7: 231-238. 